\title{
Effects of a low electron distribution cutoff on multiwavelength spectra and light curves of GRB afterglows
}

\author{
M. Petropoulou ${ }^{1}$, A. Mastichiadis ${ }^{1}$, and T. Piran $^{2}$ \\ 1 Department of Physics, University of Athens, Panepistimiopolis, 15783 Zografos, Greece \\ e-mail: maroulaaki@gmail.com \\ 2 The Racah Institute of Physics, The Hebrew University, Jerusalem, Israel
}

Received 6 November 2010 / Accepted 29 April 2011

ABSTRACT

\begin{abstract}
Aims. We investigate the behavior of the frequency-centered light curves expected within the standard model of gamma ray bursts, allowing the maximum electron energy $\left(\gamma_{\max }\right)$ to be a free parameter that may take low values.

Methods. We solve the spatially averaged kinetic equations that describe the simultaneous evolution of particles and photons, obtaining the multi-wavelength spectra as a function of time. From these we construct the frequency-centered light curves with an emphasis on the X-ray and optical bands.

Results. We show that in cases where $\gamma_{\max }$ takes low values, the produced X-ray light curves show a plateau as the synchrotron component gives its place to the synchrotron self-Compton one in the X-ray band.
\end{abstract}

Key words. gamma-rays: general - acceleration of particles - radiation mechanisms: non-thermal

\section{Introduction}

Gamma-ray bursts (GRBs) are attributed to a release of a very large amount of energy $\left(\sim 10^{51}-10^{52} \mathrm{erg}\right)$ into a small region of space $(\lesssim 100 \mathrm{~km})$ over a short period of time $\left(\sim 10-10^{2} \mathrm{~s}\right.$ for long GRBs and $\lesssim 2 \mathrm{~s}$ for short GRBs). These energetic events have two characteristic radiative signatures: (i) the prompt $\gamma$-ray and (ii) the afterglow emission. The detection of high-energy photons $\left(\epsilon_{\gamma} \gg 1 \mathrm{MeV}\right)$ implies sources of radiation that move at relativistic speeds with Lorentz factors $\Gamma$ exceeding 100 (Fenimore et al. 1993; Lithwick \& Sari 2001; Piran 1999).

While many questions concerning the prompt emission are still open, the afterglow, i.e., the lower energy long-lasting emission, is believed to arise from the interaction of the relativistic ejecta with the ambient matter and can be adequately described by the so-called "standard" model (Rees \& Mészáros 1992; Paczynski \& Rhoads 1993; Mészáros \& Rees 1997). According to this, the relativistic blast wave produced from the explosion can energize the external medium, i.e., accelerate electrons (and possibly protons) to high energies, and generate magnetic fields. The relativistic electrons radiate by synchrotron and inverse Compton radiation which is essentially the observed afterglow emission. However, to calculate the radiated photon spectra, one needs a detailed prescription of the electron distribution function and of the magnetic field. This is usually done by postulating that the electrons have a power law distribution between a minimum $\left(\gamma_{\min }\right)$ and a maximum $\left(\gamma_{\max }\right)$ cutoff with an overall energy density content that is a a fixed fraction (usually denoted by $\epsilon_{\mathrm{e}}$ ) of the total post-shock internal energy density $U$, while an analogous argument can be made for the magnetic field energy that takes a fraction $\epsilon_{\mathrm{B}}$ of $U$. A significant amount of work has been performed by many researchers in calculating the multiwavelength spectra and light curves of GRB afterglows either directly based on the above prescription (Dermer \& Chiang 1998; Sari et al. 1998; Panaitescu \& Mészáros 1998; Wijers \& Galama 1999; Dermer et al. 2000; Panaitescu \& Kumar 2000; Granot \& Sari 2002) or using different variations
(Granot \& Kumar 2006; Fan \& Piran 2006; Panaitescu et al. 2006; Zhang et al. 2006; Nousek et al. 2006).

In the present paper we focus on the effects that a low $\gamma_{\max }$ will have on the multiwavelength spectra and light curves of the afterglow emission. This has not been treated as yet because it is implicitly assumed that $\gamma_{\max }$ is very large and its radiative signature does not contribute to any observable band. However, if it has a low value, a break might appear successively in various energy bands of diminishing energy as the synchrotron component gradually cedes its position to the SSC. This will produce light curves that are not anymore pure power laws but have more complicated shapes.

The paper is structured as follows. In Sect. 2 we describe the principles of the model and discuss in a qualitative way some of the results. In Sect. 3 we quantify this and derive some analytical relations between the initial parameters, which, when satisfied, will produce different types of X-ray light curves. In Sect. 4 we make a tentative connection of our results to observations. Finally we conclude in Sect. 5 and give a brief discussion of the basic points of the present work.

\section{The model}

\subsection{First principles}

The general framework of the model we present here is based on the standard GRB afterglow model, albeit with some modifications mainly regarding the approach to the physical problem (Fan et al. 2008; Petropoulou \& Mastichiadis 2009, henceforth PM09). For the sake of completeness we recall its basic premises: as the relativistic blast wave (RBW) usually associated with GRB afterglows is expanding, it accelerates electrons of the circumstellar medium to high energies by some unspecified mechanism. These electrons are assumed to be injected behind the shock front in a volume of radius $R$ containing a tangled magnetic field $\boldsymbol{B}$. The particles suffer radiative and adiabatic 
losses, evolving with radius. At the same time they emit radiation by synchrotron and inverse Compton radiation. Therefore, there is a coupling between electrons and photons at each radius, in the sense that the electron distribution function determines the photon spectrum and, at the same time, the photons determine the electron distribution function through inverse Compton losses and, possibly, pair reinjection. The usual procedure of approaching the problem is to solve simultaneously two coupled kinetic equations for the distribution functions of electrons and photons which, when solved, give the aforementioned quantities as functions of radius and energy. The most relevant physical processes that are included are: electron synchrotron radiation, synchrotron self-absorption, inverse Compton scattering (both in the Thomson and Klein-Nishina regimes), photon-photon pair production and adiabatic losses (for a more detailed discussion regarding the physical processes see Mastichiadis \& Kirk 1995; Pe'er \& Waxman 2004).

To obtain the multiwavelength (MW) spectrum of GRBs at each radius $r$ of the relativistic blast wave, one needs to specify the Lorentz factor of the flow $\Gamma(r)$, the comoving radius of the source $R=r / \Gamma$, the magnetic field strength $B(r)-$ determined indirectly through the parameter $\epsilon_{\mathrm{B}}$, and three parameters related to the electron injection, i.e., their total power - determined by the parameter $\epsilon_{\mathrm{e}}$, their slope $p$ of the power law at injection and the maximum cutoff of their distribution $\gamma_{\max }-$ the minimum cutoff $\gamma_{\min }$ is defined in terms of the other parameters by Eq. (11).

\subsection{Multiwavelength spectra}

The approach described above allows one to calculate the photon MW spectra self-consistently while it inherently addresses questions about whether the electrons are in the fast or slow cooling regime. Moreover, it can calculate the spectrum in various regimes without resorting to a piecewise succession of broken power laws, which becomes problematic if the characteristic frequencies are fairly close to one another. Finally, it takes into account SSC losses which, as PM09 have shown - see also Sari \& Esin (2001), can significantly alter the electron spectrum and therefore, the photon spectrum, even in the well-studied synchrotron regime.

Some of the above is exemplified in Fig. 1, which depicts a generic case of a MW spectrum for a power law electron injection with $\gamma_{\max }$ not much greater than $\gamma_{\min }$. Because the parameters were chosen so that the radiating electrons are partly in the uncooled regime, the synchrotron component consists, at least in theory (Sari et al. 1998), of four segments: (1) synchrotron selfabsorbed part below $v_{\mathrm{ssa}}$; (2) typical synchrotron low-energy part for $v_{\mathrm{ssa}}<v<v_{\mathrm{s}, \min }$; (3) uncooled part for $v_{\mathrm{s}, \min }<v<v_{\mathrm{s}, \mathrm{c}}$ and (4) cooled part for $v_{\mathrm{s}, \mathrm{c}}<v<v_{\mathrm{s} \text {,max }}$. One could add here (5) the synchrotron exponential cutoff that appears above $v_{\mathrm{s}, \max }$. Between these segments power laws of different slopes should appear, but because of the proximity of the lower and upper electron cutoffs, the turnovers in frequency have been smeared out and the spectrum appears to curve continuously without any clearly defined power law regime, at least for frequencies above $v_{\mathrm{s} \text {, min }}$.

The same holds for the SSC component: this is much broader, as expected, than the synchrotron one and it also shows a continuous curvature. In Fig. 1 three characteristic frequencies of the SSC component are indicated: $v_{\mathrm{ssc}, \min }=\frac{4}{3} \gamma_{\min }^{2} v_{\mathrm{s}, \min }$, $v_{\mathrm{ssc}, \mathrm{c}}=\frac{4}{3} \gamma_{\mathrm{c}}^{2} v_{\mathrm{s}, \mathrm{c}}$ and the peak frequency $v_{\mathrm{p}}$. Sari \& Esin (2001) have shown analytically that the peak frequency of the SSC component in the slow cooling regime is given by $v_{\mathrm{ssc}, \mathrm{c}}$. However,

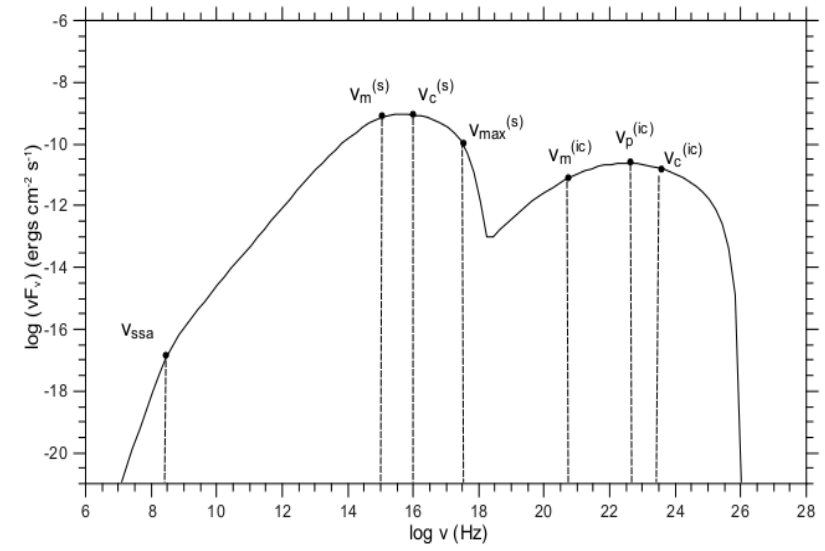

Fig. 1. Multiwavelength spectrum expected for a case where the upper electron cutoff is not much greater than the lower one - in the present case $\gamma_{\max }=10^{4}$ and $\gamma_{\min , 0}=5.6 \times 10^{3}$. The complete set of the parameters used is: $E_{0}=10^{54} \mathrm{ergs}, \Gamma_{0}=400, n_{0}=1 \mathrm{part} / \mathrm{cm}^{3}, \epsilon_{\mathrm{B}}=0.005$, $\epsilon_{\mathrm{e}}=0.01$ and $p=2.3$. Both synchrotron and SSC components of the spectrum appear to be continuously curved without any clear power law segments (at least for frequencies below $v_{\mathrm{s}, \min }$ and $v_{\mathrm{ssc} \text { min }}$ respectively) mainly because of the proximity of $\gamma_{\max }$ and $\gamma_{\min }$. The characteristic frequencies indicated in the figure are discussed in the text.

this is not expected when a relatively low $\gamma_{\max }$ is taken into account, as in the case of Fig. 1. An analytic calculation (which can be found in detail in the appendix) of the SSC peak frequency can also be made, for an electron pure power law distribution, with limits between $\gamma_{\min }$ and $\gamma_{\max }$. The SSC peak frequency is then given by $\frac{4}{3} \gamma_{\max }^{2} v_{\mathrm{s}, \min }$, which equals to $1.4 \times 10^{23} \mathrm{~Hz}$ for the example given in Fig. 1. The numerically calculated peak frequency is different, however: $v_{\mathrm{p}}=4.4 \times 10^{22} \mathrm{~Hz} \approx$ $\frac{4}{3} \gamma_{\mathrm{c}}^{2} \nu_{\mathrm{s} \text {, min }}$. This simple example shows that the presence of the Compton logarithm (Gould 1979) combined with the possibility that the electron distribution may have at least two breaks, one at $\gamma_{\min }$ and one at $\gamma_{\mathrm{c}}$, complicates a simple analytic approach. Nevertheless, for the specific example one can clearly see in $v F_{v}$ units a rising part, a broad peak and a declining part.

\subsection{Light curves}

Based on the above, it is straightforward to construct light curves at various frequencies. However, it is worth describing qualitatively a case where cutoff effects in the synchrotron component appear because of a low value of $\gamma_{\max }$. Assume a certain observing window between two frequencies. Assume also that the initial parameters are such that initially the flux in this window is dominated by the synchrotron component. As time evolves in the observer's frame, one will first observe the various parts of the synchrotron component passing through it, as was first suggested by Sari et al. (1998). Therefore, at some point in time, which we will call $t_{\mathrm{br}, 1}$, the combination of $\Gamma, B$ and $\gamma_{\max }$ will be such that the flux in the observing window will be dominated by the exponential cutoff of the synchrotron component. This will result in a natural steepening of the light curve. In the hypothetical case where the SSC component is absent, the observer would have seen an ever increasing steepening of both the spectral index and of the light curve until the flux would drop to very low levels, below the sensitivity of any instrument. In reality, however, at some level the SSC component must appear in the observing window and start dominating the flux there. This would naturally result in a flattening of the light curve because the observer starts 


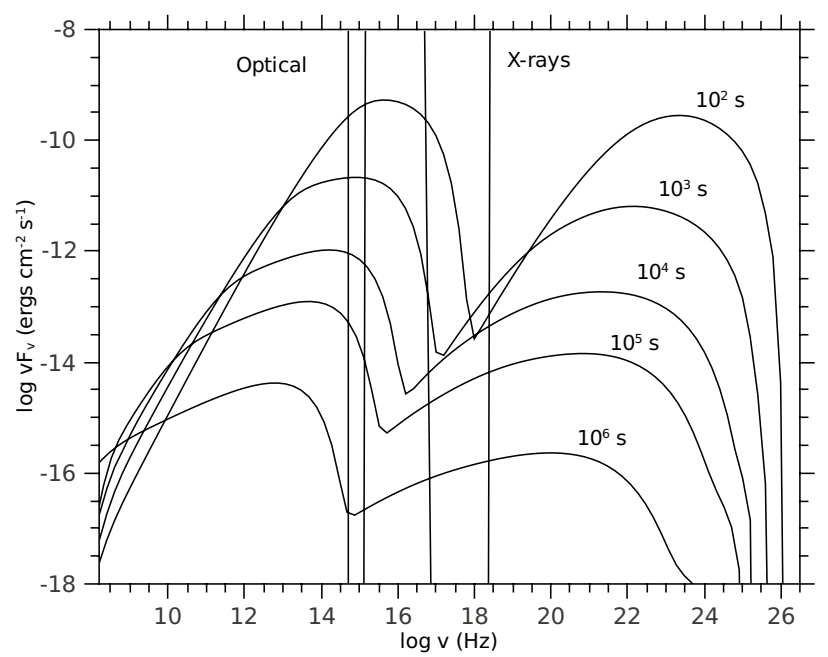

Fig. 2. Multiwavelength spectra at observer times from $10^{2} \mathrm{~s}$ to $10^{6} \mathrm{~s}$ (top to bottom). For the parameters used, see text. X-ray and optical windows that correspond to the observing energy ranges of XRT (0.3$10) \mathrm{keV}$ and UVOT (170-650) nm respectively, are also shown.

sampling photons not from the synchrotron but from the SSC component. Depending on the relation between $\gamma_{\min }$ and $\gamma_{\max }$, at time $t=t_{\mathrm{br}, 1}$ the SSC component could be, broadly speaking, in any of its three spectral regimes (i.e., rising, peak or declining). As we elaborate, below, if the SSC component becomes dominant while still early in its rising part, the light curve will show a sharp turnover, which will result in a shallow decline of the flux, i.e, a plateau. If, on the other hand, the transition from the synchrotron to SSC, occurs while the latter is close to the peak or during its declining part, the light curve will show a more gentle flattening.

Figures 2 and 3 present the results of a run showing an X-ray plateau. Figure 2 shows snapshots of the MW spectra at five different times, obtained for a GRB at $z=1$ with $E_{0}=10^{54} \mathrm{erg}$, $\Gamma_{0}=400, n_{0}=1 \mathrm{part} / \mathrm{cm}^{3}, \epsilon_{\mathrm{B}}=0.001, \epsilon_{\mathrm{e}}=0.025, p=2.3$ and $\gamma_{\max }=2.24 \times 10^{4}$. Evidently, at early times the X-ray band is dominated by the tail of the synchrotron component, which in about $t \sim 10^{3} \mathrm{~s}$ has ceded its position entirely to the SSC one. This trend is repeated once more at much later times $\left(t \gtrsim 5 \times 10^{5} \mathrm{~s}\right)$ in the optical band. Figure 3 shows the corresponding optical and X-ray light curves. The latter shows a clear plateau that gradually steepens because of the concavity of the SSC component. On the other hand, the optical light curve shows the "standard" unbroken power law behavior until $t \sim 5 \times 10^{5} \mathrm{~s}$ and flattens off slightly at even later times, as the SSC component takes over.

The above results show that if the X-ray light curves are to show plateaus, at least within the context of our model, the SSC component should be fairly flat in the X-ray energy range at the time the synchrotron component decays because of its exponential cutoff. Because the above conditions implicate the magnetic field $B(r)$, and the lower and upper cutoffs of the electron injection, $\gamma_{\min }$ and $\gamma_{\max }$ respectively, one can quantify the above conditions using the parameters of the standard afterglow model. We proceed to do this in the next section.

\section{Effects of $\gamma_{\max }$}

The upper limit of the electron distribution $\gamma_{\max }$ has not been so far considered in GRB afterglow models as a dynamic parameter.

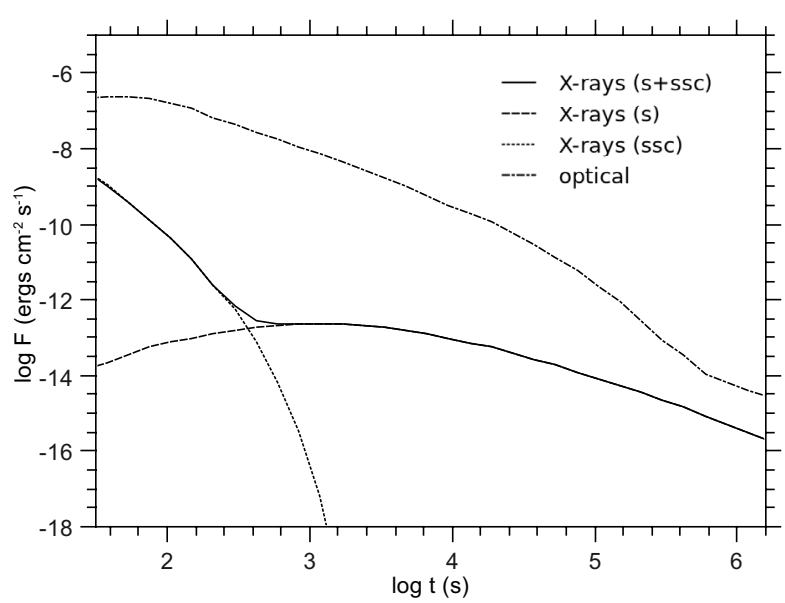

Fig. 3. X-ray (solid line) and optical (dashed-dotted line) light curve corresponding to the same case as in Fig. 2. The contribution of the synchrotron (dotted line) and SSC (dashed line) components to the total X-ray flux are also shown. The optical light curve is shifted by +2.5 units in logarithm for reasons of better display.

In this section we will show how a relatively low $\gamma_{\max }$ can affect the MW spectra and the corresponding X-ray and optical light curves. We assume that the afterglow is produced by an adiabatic relativistic blast wave that decelerates while interacting with the ISM. During this phase the evolution of the RBW is described by the self-similar solution of Blandford \& McKee (1976):

$\Gamma(r)=\Gamma_{0}\left(\frac{r}{R_{\mathrm{d}}}\right)^{-3 / 2} \quad$ if $r>R_{\mathrm{d}}$,

where

$R_{\mathrm{d}}=\left(\frac{3 E_{0}}{4 \pi n_{0} m_{\mathrm{p}} c^{2} \Gamma_{0}^{2}}\right)^{1 / 3}$.

Using the relation

$\int_{t_{0}}^{t} \mathrm{dt}=\int_{\mathrm{r}_{0}}^{\mathrm{r}} \frac{\mathrm{dr} \mathrm{r}^{\prime}}{2 \mathrm{c} \Gamma^{2}\left(\mathrm{r}^{\prime}\right)}$

and neglecting the term $t_{\mathrm{d}}=\frac{R_{\mathrm{d}}}{2 c \Gamma_{0}^{2}}$ compared to $\int_{R_{\mathrm{d}}}^{r} \frac{\mathrm{dr}{ }^{\prime}}{2 c \Gamma^{2}\left(r^{\prime}\right)}$, we find that

$r \approx\left(8 c t \Gamma_{0}^{2} R_{\mathrm{d}}^{3}\right)^{1 / 4}$.

For the strength of the magnetic field (in the fluid frame), we adopt the usual form

$B(r)=\sqrt{32 \pi n_{0} m_{\mathrm{p}} \epsilon_{\mathrm{B}}} c \Gamma(r)$.

The observed synchrotron frequency corresponding to $\gamma_{\max }$ is given by

$$
\begin{aligned}
v_{\mathrm{s}, \max } & =\Gamma \frac{e B}{2 \pi m_{\mathrm{e}} c} \gamma_{\max }^{2} \\
& =\left(\frac{3 E_{0} n_{0} m_{\mathrm{p}} \epsilon_{\mathrm{B}}^{2} e^{4}}{32 \pi^{3} c^{5} m_{\mathrm{e}}^{4}}\right)^{1 / 4} \gamma_{\max }^{2} t^{-3 / 4}
\end{aligned}
$$

We consider an X-ray window that corresponds to the observing energy range of XRT, i.e., (0.3-10) keV. We are interested in the 
time $t_{\mathrm{coff}, \mathrm{s}}^{(\mathrm{x})}$ when photons belonging to the synchrotron exponential cutoff, which forms above $v_{\mathrm{s}, \max }$ (see Fig. 1), cross a characteristic frequency of the X-ray band, say $v_{\mathrm{x}} \approx 6.3 \times 10^{17} \mathrm{~Hz}$. Accordingly, we set $v_{\mathrm{s}, \mathrm{coff}}=A v_{\mathrm{s}, \max }$ where $A$ is a numerical factor on the order of 5-10, which determines how deep into the exponential cutoff the particular synchrotron photons are. Then the expression for $t_{\mathrm{coff}, \mathrm{s}}^{(\mathrm{x})}$ becomes

$t_{\mathrm{coff}, \mathrm{s}}^{\mathrm{x})} \approx\left(\frac{3 E_{0} n_{0} m_{\mathrm{p}} \epsilon_{\mathrm{B}}^{2} e^{4}}{32 \pi^{3} c^{5} m_{\mathrm{e}}^{4}}\right)^{1 / 3} v_{\mathrm{x}}^{-4 / 3} A^{4 / 3} \gamma_{\max }^{8 / 3}$.

Demanding that the characteristic time $t_{\mathrm{coff}, \mathrm{s}}^{(\mathrm{x})}$ lies in a time interval of the general form:

$t_{k} \leq t_{\mathrm{coff}, \mathrm{s}}^{(\mathrm{x})} \leq t_{k+1}$,

where $t_{k}=10^{k} \mathrm{~s}$, Eqs. (7) and (8) combine to give the first constraining relation for $\gamma_{\max }$ :

$g_{1}\left(E_{0}, n_{0}, \epsilon_{\mathrm{B}}\right) \leq \gamma_{\max } \leq g_{2}\left(E_{0}, n_{0}, \epsilon_{\mathrm{B}}\right)$,

where $g_{1,2}\left(E_{0}, n_{0}, \epsilon_{\mathrm{B}}\right)=C_{1,2} 10^{3 k / 8} A^{-1 / 2} E_{0,54}^{-1 / 8} n_{0,0}^{-1 / 8} \epsilon_{\mathrm{B},-2}^{-1 / 4}$ with $C_{1}=4.1 \times 10^{3}$ and $C_{2}=9.7 \times 10^{3}$. One should keep in mind that relation (8) is valid only for values of the real variable $k$, which ensures that time $t_{k}$ is larger than the deceleration time $t_{\mathrm{d}}$. Here and throughout the text, the convention $Q_{x} \equiv Q / 10^{x}$ was adopted in cgs units. It is worth noting that for typical values of $E_{0,54}=n_{0,0}=\epsilon_{\mathrm{B},-2}=1$ and $k=2$ the maximum electron Lorentz factor lies between $8.1 \times 10^{3}$ and $1.9 \times 10^{4}$, which is relatively low. Although the above double inequality involves three free parameters of the model, the dependence on two of them, i.e., on $E_{0}$ and $n_{0}$, is very weak.

The SSC component peaks at a characteristic frequency that depends on the Compton logarithm first introduced by Gould (1979). In the simple case where the electron distribution is given by a pure power law between $\gamma_{\min }$ and $\gamma_{\max }$ and the scatterings happen in the Thomson regime, we found that the peak frequency of the SSC component is $v_{\mathrm{p}}=\frac{4}{3} \gamma_{\max }^{2} \nu_{\mathrm{s}, \min }$ (see appendix for a detailed calculation). In a generic case where the electron distribution shows a cooling break, the calculation of the SSC peak frequency is more complicated (see discussion in Sect. 2.2). However, in all cases the minimum frequency of the main SSC branch, i.e., $v_{\mathrm{ssc} \text {, min }}=\frac{4}{3} \gamma_{\min }^{2} v_{\mathrm{s} \text {, min }}$ is below (slow cooling regime) or at least equal (fast cooling regime) to the peak frequency. For that reason we choose to quantify the lower en-

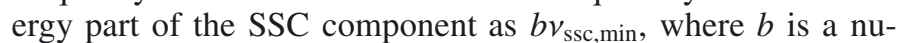
merical factor of order 0.001-0.01. In order to proceed, we need to use an expression for $\gamma_{\min }$. So far in GRB afterglow models, $\gamma_{\max }$ was not treated as a "dynamic" parameter in the sense that its signature would not be observed in the X-ray energy range and below, as it was taken to be much larger than $\gamma_{\min }$. For that reason the approximate expression for $\gamma_{\min }$ (Sari et al. 1998)

$\gamma_{\min }^{\text {approx }}=\epsilon_{\mathrm{e}} \frac{m_{\mathrm{p}}}{m_{\mathrm{e}}} \frac{p-2}{p-1} \Gamma(r)$

was safely used. However, where we also examine cases with $\gamma_{\max }$ only a few times greater than $\gamma_{\min }$, we use the accurate expression in the numerical code, which is the solution to the equation

$\frac{\gamma_{\min }^{2-p}-\gamma_{\max }^{2-p}}{\gamma_{\min }^{1-p}-\gamma_{\max }^{1-p}}=\epsilon_{\mathrm{e}} f_{p} \frac{m_{\mathrm{p}}}{m_{\mathrm{e}}} \Gamma(r)$, where $f_{p}=\frac{p-2}{p-1}$. Because the solution to this equation has no explicit analytical form, we proceed first to find a constraint of $\gamma_{\max }$ using the approximate analytical expression given by Eq. (10). For the low energy part of the SSC component we can now write

$$
\begin{aligned}
b v_{\mathrm{ssc}, \min } \approx & \frac{2 e b}{3 \pi m_{\mathrm{e}}} \sqrt{32 \pi n_{0} m_{\mathrm{p}} \epsilon_{\mathrm{B}}}\left(\epsilon_{\mathrm{e}} \frac{m_{\mathrm{p}}}{m_{\mathrm{e}}} f_{p}\right)^{4} \\
& \times\left(\frac{3 E_{0}}{2048 \pi n_{0} m_{\mathrm{p}} c^{5}}\right)^{3 / 4} t^{-9 / 4}
\end{aligned}
$$

In order to have a flattening of the light curve after the first break, this low-energy part of the SSC component must appear in the $\mathrm{X}$-ray band about the time when the synchrotron one decays. In complete analogy to $t_{\text {coff, } \mathrm{s}}^{(\mathrm{x})}$, the observed time $t_{\mathrm{ssc}}^{(\mathrm{x})}$ at which the frequency $b v_{\mathrm{ssc}}$, min enters the X-ray band is determined by the relation

$$
\begin{aligned}
t_{\mathrm{ssc}}^{\mathrm{x})}= & v_{\mathrm{x}}^{-4 / 9}\left(\frac{2 e b}{3 \pi m_{\mathrm{e}}}\right)^{4 / 9}\left(32 \pi n_{0} m_{\mathrm{p}} \epsilon_{\mathrm{B}}\right)^{2 / 9}\left(\epsilon_{\mathrm{e}} \frac{m_{\mathrm{p}}}{m_{\mathrm{e}}} f_{p}\right)^{16 / 9} . \\
& \left(\frac{3 E_{0}}{2048 \pi n_{0} m_{\mathrm{p}} c^{5}}\right)^{1 / 3} .
\end{aligned}
$$

The ratio of the two characteristic times is given by

$$
\begin{aligned}
\frac{t_{\mathrm{ssc}}^{(\mathrm{x})}}{t_{\mathrm{coff}, \mathrm{s}}^{(\mathrm{x})}} & =\left[\left(v_{\mathrm{x}} \frac{m_{\mathrm{p}}}{m_{\mathrm{e}}}\right)^{2} \frac{\pi m_{\mathrm{p}}}{6 e^{2}}\right]^{4 / 9} b^{4 / 9} A^{-4 / 3} \gamma_{\max }^{-8 / 3}\left(\frac{f_{p}^{4} \epsilon_{\mathrm{e}}^{4}}{n_{0} \epsilon_{\mathrm{B}}}\right)^{4 / 9} \\
& \approx 2 \times 10^{16} b^{4 / 9} A^{-4 / 3} \gamma_{\max }^{-8 / 3}\left(\frac{f_{p}^{4} \epsilon_{\mathrm{e}}^{4}}{n_{0} \epsilon_{\mathrm{B}}}\right)^{4 / 9} .
\end{aligned}
$$

When the two timescales $t_{\text {coff, } \mathrm{s}}^{(\mathrm{x})}$ and $t_{\mathrm{ssc}}^{(\mathrm{x})}$ are of the same order, a break will appear in the X-ray light curve, because the synchrotron component gives its place to the low-energy part of the SSC one. Allowing

$0.2<\frac{t_{\mathrm{ssc}}^{(\mathrm{x})}}{t_{\mathrm{coff}, \mathrm{s}}^{(\mathrm{x})}}<1.0$

we obtain one more constrain for $\gamma_{\max }$, i.e.,

$h_{1}\left(n_{0}, \epsilon_{\mathrm{e}}, \epsilon_{\mathrm{B}}, f_{p}\right)<\gamma_{\max }<h_{2}\left(n_{0}, \epsilon_{\mathrm{e}}, \epsilon_{\mathrm{B}}, f_{p}\right)$,

where the functions $h_{1,2}$ are defined as $h_{1,2}=$ $K_{1,2} \quad A^{-1 / 2} b^{1 / 6} f_{p}^{2 / 3} \epsilon_{\mathrm{e},-1}^{2 / 3} n_{0,0}^{-1 / 6} \epsilon_{\mathrm{B},-2}^{-1 / 6}$ with $K_{1}=6 \times 10^{5}$ and $K_{2}=1.1 \times 10^{6}$. The above relations make the tacit assumption that the scatterings occur in the Thomson regime, at least for the lower energy part of the SSC spectrum. For that reason, we impose one more constraint on $\gamma_{\max }$, demanding that at least for $t \sim t_{\text {coff,s }}^{(\mathrm{x})}$ the assumption of scattering in the Thomson regime is valid. For that we use the dimensionless factor

$$
\begin{aligned}
x & =\frac{\gamma_{\min } h v_{\mathrm{s}, \min }^{\prime}}{m_{\mathrm{e}} c^{2}} \\
& =\frac{\hbar e}{8 m_{\mathrm{e}}^{2} c^{2}}\left(\frac{3 E_{0} \epsilon_{\mathrm{B}}}{c^{5}}\right)^{1 / 2}\left(f_{p} \frac{\epsilon_{\mathrm{e}} m_{\mathrm{p}}}{m_{\mathrm{e}}}\right)^{3} t^{-3 / 2},
\end{aligned}
$$

where primed quantities are measured in the comoving frame. At $t=t_{\text {coff, } \mathrm{s}}^{(\mathrm{x})}$ the $x$ parameter is given by

$x=\frac{\hbar f_{p}^{3} m_{\mathrm{p}}^{3}}{8 e m_{\mathrm{e}}^{3} c^{2}}\left(\frac{32 \pi^{3}}{m_{\mathrm{p}}}\right)^{1 / 2} v_{\mathrm{x}}^{2} \epsilon_{\mathrm{e}}^{3} A^{-2} \gamma_{\max }^{-4}\left(n_{0} \epsilon_{\mathrm{B}}\right)^{-1 / 2}$. 


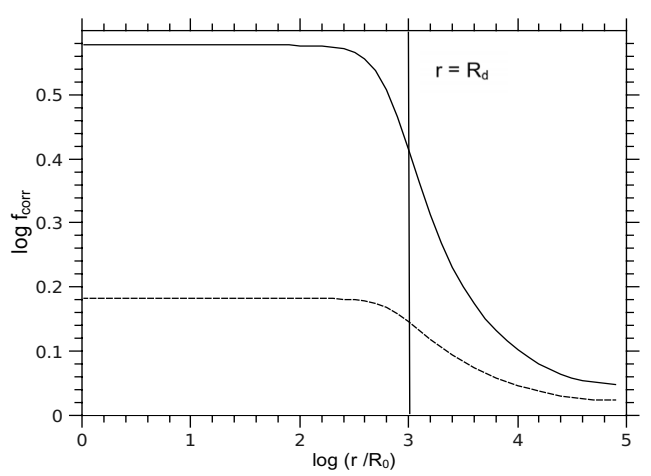

Fig. 4. Log-log plot of $f_{\text {mod }}$ as a function of radius $r$ (in units of the initial radius $R_{0}=10^{14} \mathrm{~cm}$ ) for two values of the ratio $\frac{\gamma_{\max }}{\gamma_{\min , 0}}: 1.32$ (solid line) and 33 (dashed line). Other parameters used are $E_{0}=10^{54} \mathrm{erg}$, $\Gamma_{0}=400, n_{0}=1 \mathrm{part} / \mathrm{cm}^{3}, \epsilon_{\mathrm{B}}=0.001, \epsilon_{\mathrm{e}}=0.01$ and $p=2.3$. The deceleration radius is also shown.

Thus, when $x<1$ we find that

$\gamma_{\max } \gtrsim 50 A^{-1 / 2} n_{0,0}^{-1 / 8} f_{p}^{3 / 4} \epsilon_{\mathrm{e},-1}^{3 / 4} \epsilon_{\mathrm{B},-2}^{-1 / 8}$.

The above relation for typical parameter values poses only a weak constraint on $\gamma_{\max }$. The same holds even if the accurate expression for $\gamma_{\min }$ was used. For that reason from here on we will not consider the constraining relation (20).

After determining the constraining relations for $\gamma_{\max }$ using the approximate form of $\gamma_{\min }$, we can now estimate the corrections introduced, after taking into account its accurate expression. For the purposes of our analytic analysis we model it as

$\gamma_{\min }=f_{\bmod } \gamma_{\min }^{\text {approx }}$

where the term $f_{\text {mod }}$ is a function of the radius $r$ and of the ratio $\frac{\gamma_{\max }}{\gamma_{\min , 0}} ; \gamma_{\min , 0}$ is the initial minimum Lorentz factor of the electrons. Function $f_{\text {mod }}$ is plotted, for illustrative reasons, against radius $r$ for two values of $\frac{\gamma_{\max }}{\gamma_{\min , 0}}$ in Fig. 4. Evidently, $f_{\bmod } \rightarrow 1$ in the limit of $\gamma_{\max } \gg \gamma_{\min , 0}$, as expected. Because our analysis holds for the deceleration phase of the RBW, we can simplify the calculations even more by averaging the function $f_{\text {mod }}$ over $\tilde{r}=\log r$ for $r>R_{\mathrm{d}}$ :

$\bar{f}_{\text {mod }}\left(\frac{\gamma_{\max }}{\gamma_{\min , 0}}\right)=\frac{\int_{r>R_{\mathrm{d}}} \mathrm{d} \tilde{r} f_{\bmod }\left(\tilde{r}, \frac{\gamma_{\max }}{\gamma_{\min , 0}}\right)}{\int_{r>R_{\mathrm{d}}} \mathrm{d} \tilde{r}}$.

This average modifying parameter can be used in Eq. (21) instead of $f_{\text {mod }}$. Thus, constraining relation (17) becomes

$\bar{h}_{1}<\gamma_{\max }<\bar{h}_{2}$,

where $\bar{h}_{1,2}=\bar{f}_{\text {mod }}^{2 / 3} h_{1,2}$. Table 1 shows some indicative values of the correction introduced in relation (23) for $E_{0}=10^{54} \mathrm{erg}$, $\Gamma_{0}=400, n_{0}=1 \mathrm{part} / \mathrm{cm}^{3}, \epsilon_{\mathrm{B}}=0.001 \epsilon_{\mathrm{e}}=0.01$ and $p=2.3$.

Using Eqs. (9) and (23) we can plot $\gamma_{\max }$ versus $\epsilon_{\mathrm{e}}$ for fixed values of $E_{0}, n_{0}, p$ and $\epsilon_{\mathrm{B}}$, creating a parameter space shown in Fig. 5. The other parameters used are the same as those in Table 1 . The curves defined by Eqs. (9) and (23) create distinctive areas on the parameter space. For initial values chosen from the striped area, Eq. (11) has as solution $\gamma_{\min , 0}=\gamma_{\max }$ with no physical meaning. In that sense the striped area is not permitted. The horizontal zones labeled by $k=2$ and $k=3$ are related to cases where the transition time $t_{\mathrm{coff}, \mathrm{s}}^{(\mathrm{x})}$ lies between $10^{2}-10^{3} \mathrm{~s}$ and $10^{3}-10^{4} \mathrm{~s}$ respectively. A choice of $k<2$ would correspond to
Table 1. Indicative values of the correction introduced in the constraining relation $(23)$.

\begin{tabular}{lcc}
\hline \hline $\log \gamma_{\max }$ & $\log \left(\gamma_{\max } / \gamma_{\min , 0}\right)$ & $\frac{2}{3} \log \bar{f}_{\bmod }$ \\
\hline 4.15 & 0.495 & 0.087 \\
4.35 & 0.787 & 0.072 \\
5.35 & 2.00 & 0.030 \\
\hline
\end{tabular}

Notes. For the parameters used, see text.

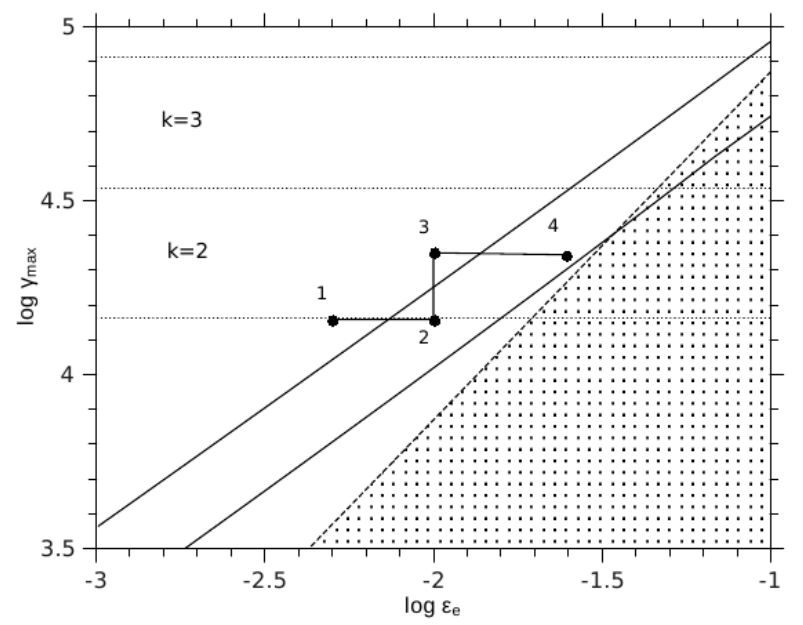

Fig. 5. Initial value parameter space of $\gamma_{\max }$ and $\epsilon_{\mathrm{e}}$ for $E_{0}=10^{54} \mathrm{erg}$, $\Gamma_{0}=400, n_{0}=1$ part $/ \mathrm{cm}^{3}, \epsilon_{\mathrm{B}}=0.001$ and $p=2.3$. The numerical factors chosen here are $A=8, b=0.001$ (see text for their definition). Dotted lines correspond to the constraining relation (9), solid lines to (23) and the dashed line sets the boundary of the striped area, which is not permitted because it it leads to $\gamma_{\max }=\gamma_{\min , 0}$. The horizontal zones labeled by $k=2$ and $k=3$ correspond to transition times $t_{\text {coff, }}^{(\mathrm{x})}$ lying in the time intervals $10^{2}-10^{3} \mathrm{~s}$ and $10^{3}-10^{4} \mathrm{~s}$, respectively. The "coordinates" $\left(\epsilon_{\mathrm{e}}, \gamma_{\max }\right)$ of the points 1 to 4 marked on the plot are $\left\{\left(0.005,1.41 \times 10^{4}\right),\left(0.01,1.41 \times 10^{4}\right),\left(0.01,2.24 \times 10^{4}\right),(0.025,2.24 \times\right.$ $\left.\left.10^{4}\right)\right\}$, respectively . The corresponding X-ray and optical light curves of the points are presented below in Figs. 6 and 8.

breaks occuring at even earlier times. We call the tilted zone a "plateau strip", because a choice of a pair $\left(\epsilon_{\mathrm{e}}, \gamma_{\max }\right)$ from it leads to X-ray light curves that show a shallow decline phase, i.e., a "plateau". Moreover, if this pair is chosen from the intersection of the "plateau strip" with a horizontal zone labeled by $k=2$ for example, the plateau phase will begin at a time between $10^{2}-$ $10^{3} \mathrm{~s}$ and so on. Finally, a choice of pairs from the area outside the tilted zone leads to X-ray light curves with a single change of slope.

For a better inspection of the parameter space, we chose pairs from four distinctive regions of the diagram and show the corresponding X-ray and optical light curves in Figs. 6 and 8. As we move from point 1 to 2 , i.e., by increasing $\epsilon_{\mathrm{e}}$ while keeping $\gamma_{\max }$ constant, the break times (see Fig. 6) remain constant while the light curve shapes exhibit a transition as the decay becomes flatter, which leads to a plateau phase. From point 2 to 3, the break is shifted toward later times while the light curve becomes once again steep. This is to be as expected because point 3 lies outside the "plateau strip". Finally, as we move from point 3 to 4, the slope of the light curve after the break becomes flat and a plateau is produced. This behavior is the same for the already discussed transition from point 1 to 2 , with the sole exception that now the break occurs at later times. Note that for all the numerical runs we present here, we use the accurate expression for 


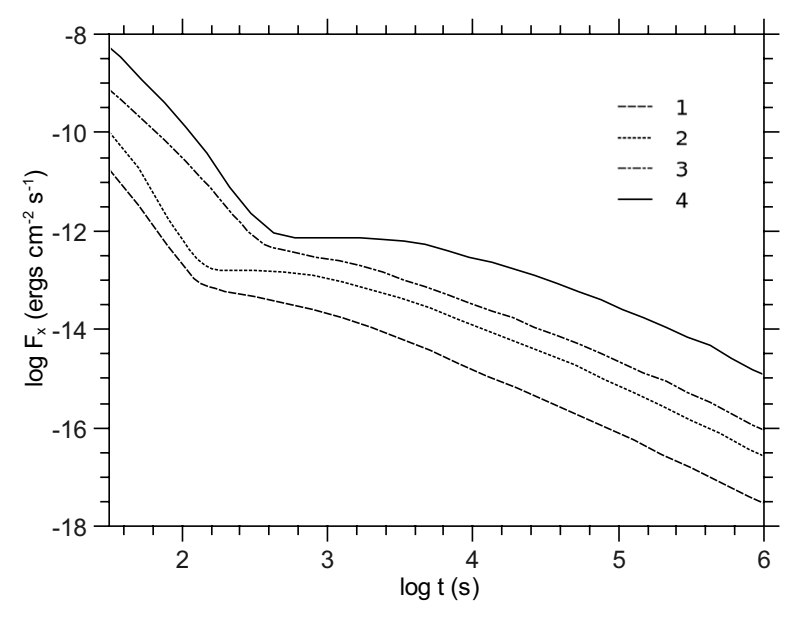

Fig. 6. X-ray light curves for different set of parameters corresponding to the points from 1 to 4 (bottom to top) marked in Fig. 5. The first break of the X-ray light curves 1 and 2 occurs at around $125 \mathrm{~s}$, while for the light curves 3 and 4 occurs later at $t \sim 650 \mathrm{~s}$. For clarity reasons, light curves 1,3 , and 4 are plotted with an offset of $-0.2,+0.4$ and +0.5 in logarithmic units of flux.

$\gamma_{\min }$ by solving Eq. (11). That is why we included a subroutine in our numerical code that utilizes a combination of the bisection and Newton-Raphson method (Press et al. 1992).

We add some comments on the effects that the other free parameters, which were assumed fixed in Fig. 5, will have on the parameter space. For example, a possible change of $E_{0}$ will only affect the dotted lines in Fig. 5. The effect will not be very significant because the inequality (9) only very weakly depends on $E_{0}$. In general, an increase of $\epsilon_{\mathrm{B}}$ will shift the "plateau strip" downward. Although the parameter $\Gamma_{0}$ does not explicitly appear in the constraining relations (9) and (23), has an effect on the relative positions of the striped area and the "plateau strip". Finally, the external number density $n_{0}$ may not severely affect the appearance of the parameter space, but has a significant effect on the ratio of the synchrotron to the SSC flux. We note that as PM09 showed, an increase of $n_{0}$ makes the afterglow more Compton dominant. This possibility has also been discussed by Panaitescu \& Kumar (2000) and Sari \& Esin (2001). A denser external medium increases in $v F_{v}$ units the X-ray flux over the optical. These high values of the external density are required by our model if the X-ray light curve shows a shallow phase and the ratio $L_{\mathrm{X}} / L_{\mathrm{opt}}>1$. Figure 7 shows such a case. The parameters used are $E_{0}=10^{54} \mathrm{erg}, \Gamma_{0}=100, n_{0}=10^{3}, \epsilon_{\mathrm{B}}=10^{-5}$, $\epsilon_{\mathrm{e}}=0.032$ and $\gamma_{\max }=8.2 \times 10^{4}$. For $t>10^{3} \mathrm{~s}$, when the spectral evolution in the X-ray energy band is not very significant, the optical flux lies approximately one order of magnitude below the $\mathrm{X}$-ray one.

As the RBW decelerates, the synchrotron and SSC emission from the forward shock becomes weaker and softer (see Fig. 2). As a result, the synchrotron cutoff will enter at some instant $t_{\text {coff,s }}^{\text {(opt }}$ in the optical band. Under the assumptions of the problem, i.e., when $B \propto r^{-3 / 2}$ and $\gamma_{\max }$ independent of $r$, it is straightforward to show that this is related to $t_{\text {coff, }}^{(\mathrm{x})}$ by

$t_{\mathrm{coff}, \mathrm{s}}^{(\mathrm{opt})}=\left(\frac{v_{\mathrm{x}}}{v_{\mathrm{opt}}}\right)^{4 / 3} t_{\mathrm{coff}, \mathrm{s}}^{(\mathrm{x})}$

or, equivalently, by

$t_{\mathrm{coff}, \mathrm{s}}^{(\mathrm{opt})} \approx 2 \times 10^{3} t_{\mathrm{coff}, \mathrm{s}}^{\mathrm{(x)}}$,
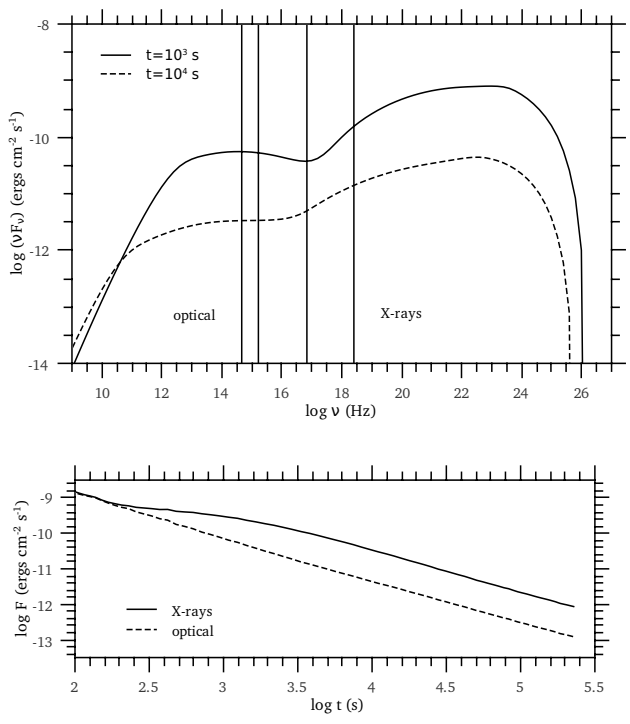

Fig. 7. Multiwavelength spectra at observer times $10^{3} \mathrm{~s}$ and $10^{4} \mathrm{~s}(t o p)$ and light curves for the X-ray and optical band (bottom) for an SSC dominated afterglow. For the parameters used, see text. The X-ray and optical windows are as given in Fig. 2.

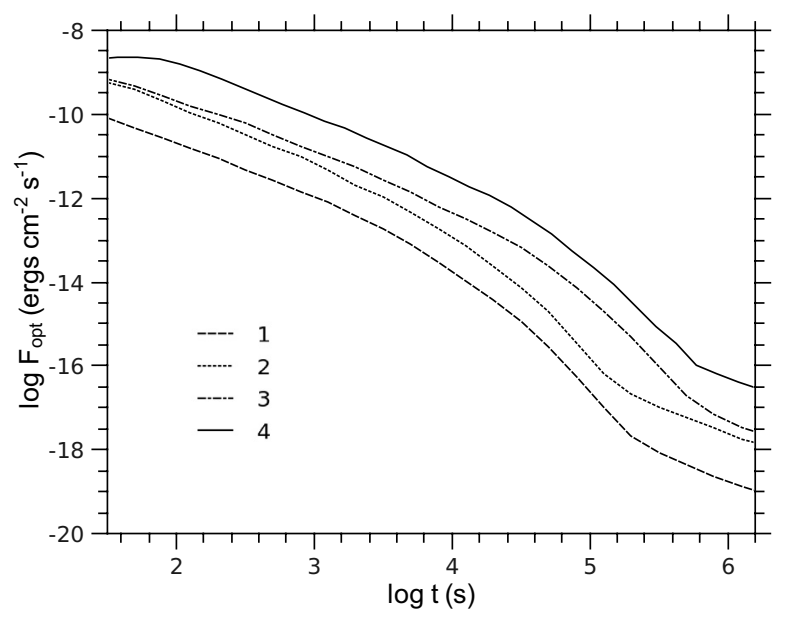

Fig. 8. Optical light curves for different sets of parameters corresponding to the points 1 to 4 (bottom to top) marked in Fig. 5. For clarity reasons, light curves 1,3 , and 4 are plotted with an offset of $-0.4,+0.2$ and +0.5 in logarithmic units of flux.

where a typical optical frequency $v_{\mathrm{opt}}=2 \times 10^{15} \mathrm{~Hz}$ was used. For the cases represented by points 1 and 2 in Fig. 5 one finds $t_{\text {coff, }}^{(\mathrm{x})} \approx 125 \mathrm{~s}$ (see also Fig. 6). Thus, a break in the optical light curve is expected at around $2.5 \times 10^{5} \mathrm{~s}$, which is confirmed numerically and is shown in Fig. 8. The other two optical light curves in the same figure show a break at correspondingly later times $\left(\gtrsim 6.3 \times 10^{5} \mathrm{~s}\right)$. We should also note that the break of the optical light curve will not neccessarily be of a "plateau" type. This is because the emerging SSC component has become steeper since it emerged in the X-ray window (see Fig. 2 for the time evolution of the SSC component). A natural outcome of our model is that the optical light curves do not exhibit a break during the plateau phase of the corresponding one in X-rays, which was first noted by Fan \& Piran (2006). Evidence for chromatic breaks in X-ray light curves were also discussed in Panaitescu et al. (2006). 


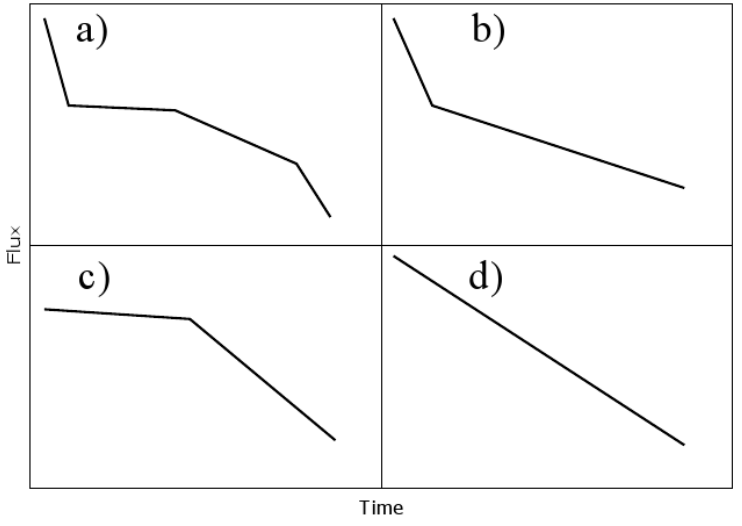

Fig. 9. Schematic diagram from Evans et al. (2009) showing the different observed light curve morphologies.

Chromatic breaks in the optical and X-ray light curves can be produced by our model with an expected time difference given by Eq. (25). As we discuss in the last section, this is a strong constraint, which reflects the validity of the model's assumptions.

\section{Basic results}

It is well known that the X-ray and optical light curves of GRB afterglows show a wide range of behaviors. Especially in the X-ray regime (Nousek et al. 2006; Evans et al. 2009), the behavior can be quite perplexing (see Fig. 9) with many cases showing a plateau that is quite difficult to interpret within the context of the standard model. The analysis performed in Sect. 3 can be tentatively relevant to these observations. Using for instance the example set by Fig. 5 as a guide, we can say that plateaus appear as long as the values of $\gamma_{\max }$ and $\epsilon_{\mathrm{e}}$ are chosen from the region defined by the two tilted lines. Moving inside this region from the lower left to the upper right, plateaus appear at progressively longer times. Furthermore, a choice of the initial parameters outside this region (for example, from the left top corner of Fig. 5) leads to afterglows without a plateau phase. Figure 10 shows different types of X-ray light curves obtained using our numerical code corresponding to points from different regions of the parameter space of Fig. 5. Light curves in panels (a) and (b) correspond to points (4) and (1) already shown in Fig. 5, while light curves of panels (c) and (d) are obtained using $\left(\epsilon_{\mathrm{e}}, \gamma_{\max }\right)=\left(0.0032,6.3 \times 10^{3}\right)$ and $\left(0.01,10^{6}\right)$, respectively ${ }^{1}$. A tentative comparison of our model light curves to those of Fig. 9 can be made.

Because the model presented here produces multiwavelength spectra at each instant, we can use it to calculate the evolution of the expected X-ray hardness ratio defined as the ratio of counts in the $1.5-10 \mathrm{keV}$ to the counts in the $0.1-1.5 \mathrm{keV}$ band (Evans et al. 2009, 2010). Figure 11 shows the time evolution of the hardness ratio for each of the example cases shown in Fig. 10. Time evolution of the corresponding photon index is also shown in the inserts of Fig. 11, whenever the spectral shape allows its viable calculation at the particular time (for a more detailed discussion on the shape of our X-ray model spectra see Sect. 2).

For X-ray light curves with a distinctive "plateau" phase, we find that the spectral evolution shows a characteristic trend as the $\mathrm{X}$-ray window is first dominated by the synchrotron and later by the SSC component. This can be seen in panel (a) of Fig. 11. At

\footnotetext{
${ }_{1}$ All other parameters used are the same as in Fig. 5 except for the case presented in panel (c), where $\Gamma_{0}=800$ was used.
}
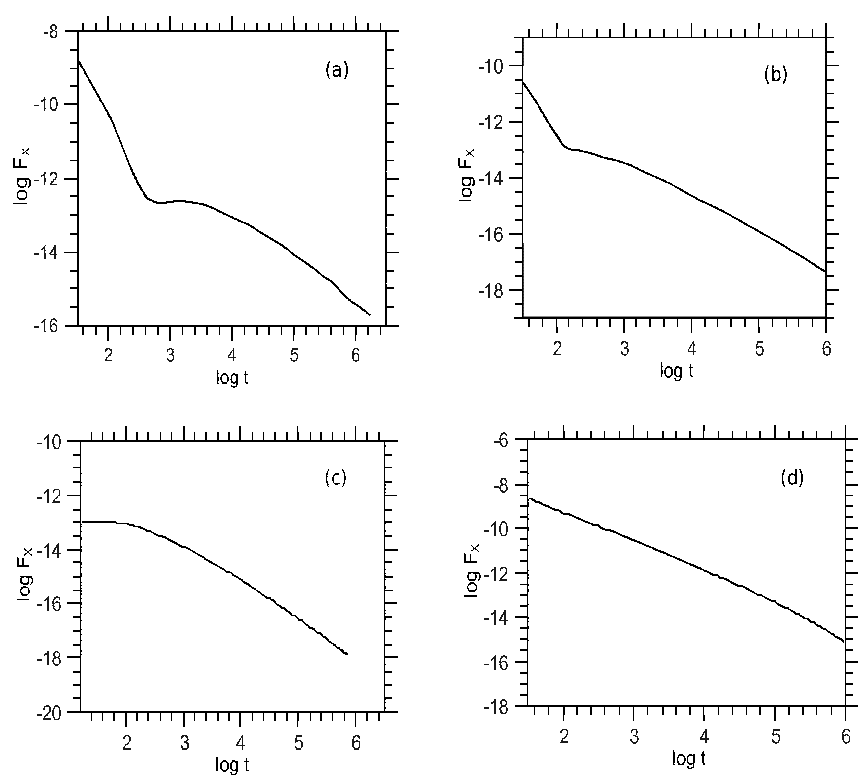

Fig. 10. Different light curve morphologies obtained using our numerical code corresponding to points from different regions of the parameter space shown in Fig. 5. Light curves from each panel can be tentatively compared to the corresponding ones of Fig. 9. For the parameters used, see text.
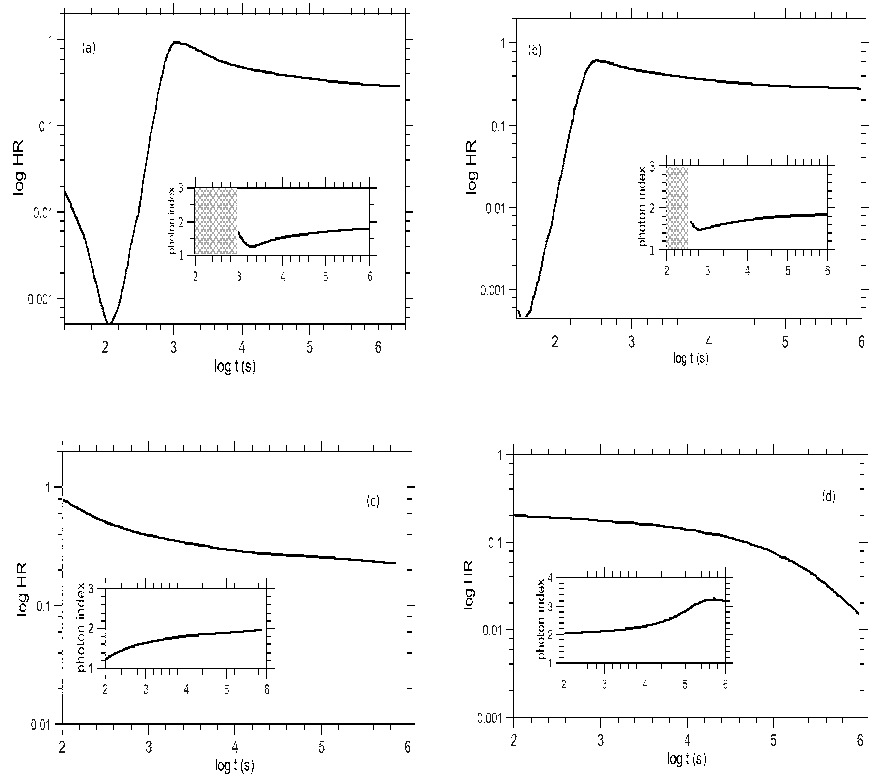

Fig. 11. Time evolution of the hardness ratio for each of the cases presented in Fig. 10. For the definition of the used hardness ratio, see text. The insert in each panel shows the evolution of the corresponding photon index. The shaded areas in the inserts imply that during this period our model spectra cannot be approximated by a single power law, and therefore a specific photon index could not be attributed to the spectrum.

very early times both the soft and hard X-ray bands are dominated by the synchrotron photons - however, the hard band is affected first by the synchrotron cutoff and this has as a result the decrease of the hardness ratio. During this phase the spectrum in X-rays is shaped by an exponential cutoff (synchrotron emission) and an emerging flat power law component (SSC emission). Thus, it cannot be simply approximated by a single power law and "labeled" by a photon index (shaded area in the insert). 
At later times, the SSC component starts appearing in the hard band while the decreasing synchrotron component dominates the soft one, resulting in an increase of the hardness ratio. Finally, at even later times both bands are dominated by the SSC component, whose low-energy part can be approximated by a flat power law, and because of its gradual steepening the hardness ratio appears to decrease gently.

If the X-ray flux decays as a power law with time, as in panel (d) of Fig. 10, we find no significant spectral evolution. The photon index is approximately constant almost for three or four decades in time, because the power law segment of the synchrotron component dominates until late times in the X-rays (see insert in panel (d) of Fig. 11). The other two cases presented in panels (b) and (c) of Fig. 11 lie somewhere in between the two aforrementioned example cases.

Although the qualitative evolution of the photon index with time is a robust feature of our model, its specific value depends on the value of the other model parameters, such as the slope of the electron energy spectrum. In all our runs we used a typical value of $p=2.3$.

\section{Summary and discussion}

We investigated the role that the upper cutoff of the electron injection can play in the evolution of the multiwavelength spectra and light curves of GRB afterglows. For this we solved selfconsistently the kinetic equations that govern the electron evolution and photon radiation as a function of distance (see also Fan et al. 2008, and PM09). This approach can successfully address the effects of the electron cutoff radiation on the light curves.

We showed that depending on the adopted value of $\gamma_{\max }$ the $\mathrm{X}$-ray lightcurves can exhibit one of the following behaviors:

1. If $\gamma_{\max }$ is not much greater than the lower cutoff $\gamma_{\min }$, the $\mathrm{X}$-ray light curves show three distinct phases. First a fast drop phase, which corresponds to the exponential cutoff of the synchrotron component. Then a plateau phase, which is caused from the gradual dominance of the SSC component over the decaying synchrotron. Finally, a more gradual power law decay, which corresponds to the normal evolution of the SSC component. The analytical approach used in Sect. 3 and summarized in Fig. 5 shows that depending on the initial parameters, there might be a narrow strip in phase space that produces $\mathrm{X}$-ray plateaus. In all cases we found that $\gamma_{\max }$ should be no more than a factor of 10 larger than $\gamma_{\min }$.

2. If $\gamma_{\max }$ does not satisfy the above condition but is still close to the "plateau strip", then the X-ray afterglows do not show a plateau but simply a change in the power law decay, i.e., the three phases degenerate into two.

3. If $\gamma_{\max } \gg \gamma_{\min }$, we find that the X-ray afterglows will be dominated until very late times by the synchrotron component, i.e., we obtain the standard afterglow picture.

These trends are exemplified in Figs. 6 and 10. For the flux decay in the optical regime, the present model predicts that the optical light curve will mimic the X-ray one with two major differences (see Fig. 8). First, the break in the optical light curve will occur at much later times (see Eq. (25)) and second, after the break, the optical light curve will not neccessarily show a plateau. Note, however, that both of these statements are based on the assumption that $\gamma_{\max }$ remains constant throughout the RBW evolution.

In this respect, $\gamma_{\max }$ emerges as one of the important parameters of the afterglow evolution because its choice can control critically the behavior of the X-ray light curves.
The evolution of the X-ray hardness ratio and spectral indices were presented in Fig. 11. Our model-derived hardness ratio shows a characteristic signature that is compatible with observations at least during the early stages (Butler \& Kocevski 2007; Liang et al. 2007). On the other hand, it is still inconclusive regarding the late stages (P. O'Brien - priv. comm.), because the transition from the plateau to the normal decay shows an evolution in the hardness ratio by a factor of two, which is not the case for several individual GRBs (e.g. Vaughan et al. 2006; Liang et al. 2007). However, as preliminary calculations have shown (Petropoulou et al. 2011 - to appear in the proceedings of the 25th Texas Symposium held last December in Heidelberg), this is greatly relaxed if $\gamma_{\max }$ is allowed to increase with radius. Because the study of such cases is beyond the scope of the present paper, we will treat this in a forthcoming publication.

It is important to emphasize at this point that the close relation we found between X-ray light curves that exhibit a plateau phase and a $\gamma_{\max }$ not much greater than $\gamma_{\min }$, is what actually distinguishes our model from other works (e.g. long-lasting energy injection into the forward shock - refreshed shock models (Zhang et al. 2006), late prompt emission (Ghisellini et al. 2007; Ghisellini 2008), geometrical effects (Eichler \& Granot 2006; Granot et al. 2006), nonstandard deceleration of the bulk Lorentz factor owning to the Compton-drag force (Mastichiadis \& Kazanas 2009), dust scattering (Shao et al. 2008, and others). Some recent PIC simulations show that most of the particles are accelerated into a relativistic Maxwellian, while a small fraction of them is injected in a power law high-energy tail, whose high-energy cutoff is approximately only one order of magnitude larger than the low one (Spitkovsky 2008; Sironi \& Spitkovsky 2009). However, this is not a final result, because the present numerical simulations have not reached yet a steady state, where $\gamma_{\max }$ is expected to be larger than the one found so far. It is also interesting to note that when we set $\gamma_{\max }$ not much larger than $\gamma_{\min }$ the qualitative behavior of our results is similar to the one obtained when one replaces the power law injection with a relativistic Maxwellian (Giannios \& Spitkovsky 2009). All other parameters used in our work are the same as in the standard GRB afterglow model.

Spectral evolution during the steep early phase, which is observed in a significant number of GRB afterglows (see Zhang et al. 2007, for a systematic study of 44 steeply decaying X-ray afterglows), is an inevitable outcome of our model, because in our present work the early steep decay of the X-ray light curve is atrributed to the emission from the external shock. Moreover, there is some observational evidence for a possible smooth connection of the early afterglow to the prompt emission (Barthelmy et al. 2005). In that case the present work could be regarded as an extension of the supercritical model (Mastichiadis \& Kazanas 2009) to the afterglow regime. Alternatively, it can result from a gradual transition toward the end of the prompt phase to an external shock emission. Evidence for this transition is seen in several examples where the extrapolated BAT light curve is not connected to early-time XRT light curve (Tagliaferri et al. 2005). This combined with a strong spectral evolution at early times suggests that the two emissions are produced by either different mechanisms or in different regions. Such a transition from the prompt to the afterglow emission could be explained within the context of the internal/external shock scenario (Piran 1999, 2004 - see also Dermer 2007, for a discussion on the possible scenarios). 
Finally, we would like to present potentially problematic points of our model - some of these could be addressed with future observations and further analysis.

1. For an afterglow with a plateau phase we find that the X-ray spectrum cannot be represented by a simple power law during the steep decay-early plateau phase. The spectral shape at this stage can more accurately be described by a steep component coming from the synchrotron cutoff plus a flat power law coming from the emerging SSC component - see Fig. 2 at early times.

2. Some preliminary efforts in fitting lightcurves and hardness ratios $^{2}$ have shown that in some cases, our model can successfully reproduce both (e.g. GRB 060512 - see Petropoulou et al. 2011). However, in other cases the model can successfully fit only the lightcurve, while the HR fit is poorer (e.g. GRB 050713B).

3. If the light curve shows an abrupt break (either a plateau phase or a single change in the slope decay) and $L_{X} / L_{\text {opt }}>1$, then our model requires high values of the external density $\left(n \geq 100 \mathrm{~cm}^{-3}\right)$. This could be problematic in cases of GRB afterglows for which low values of $N_{\mathrm{H}}$ are derived (Schady et al. 2007). We note, however, that in all other cases the above constraint of our model is relaxed.

4. For those GRBs where the early steep decay is smoothly connected to the prompt emission phase, the model requires that the late prompt emission is already dominated by an external shock emission. This arises naturally in the external shock model or it requires a transition during the late prompt phase from in the internal to external shock.

Concluding, we remark that the consideration of the electron distribution's upper energy cutoff as another free parameter in the standard afterglow model brings many interesting features in the light curve/spectral behavior of GRB afterglows. All these are related to the evolution of the relativistic electron distribution and eventually point out the acceleration mechanism at work. We plan to deal with this question in a forthcoming publication.

Acknowledgements. A.M. would like to thank T.P. for hospitality during a visit of the Hebrew University. We thank Drs. P. Evans, P. O'Brien and A. Zezas for useful discussions and Dr. D. Giannios for comments on the manuscript. This work made use of data supplied by the UK Swift Science Data Centre at the University of Leicester. This research has been co-financed by the European Union (European Social Fund - ESF) and Greek national funds through the operational Program "Education and Lifelong Learning" of NSRF - Research Funding Program: Heracleitus II.

\section{Appendix A: Synchrotron self-Compton spectrum of a power law electron distribution}

We assume a spherically symmetric source of radius $R$ with a magnetic field $B$ randomly oriented. Synchrotron radiation is produced by an isotropic electron distribution of the form

$n_{\mathrm{e}}=k_{\mathrm{e}} \gamma^{-p} \quad \gamma_{\min }<\gamma<\gamma_{\max }$

where $p$ is the exponent of the power law spectrum, $\gamma_{\min }, \gamma_{\max }$ are the cutoffs of the distribution and $k_{\mathrm{e}}$ is a normalization factor that determines the electron density and depends on radius $r$ for

\footnotetext{
2 Although a successful fit of the HR could not neccessarily mean a succesful spectral fit, we preferred to model the former because in many cases our X-ray spectra cannot be reproduced well by a single power law - see also the previous point.
}

an inhomogeneous source. The synchrotron photon production rate per unit energy and per unit volume is then given by

$\frac{\mathrm{d} N_{\mathrm{s}}}{\mathrm{d} \epsilon \mathrm{d} t}=\frac{2 e^{3}}{m_{\mathrm{e}} h^{2} c^{2}}\left(\frac{3 e}{4 \pi m_{\mathrm{e}} c}\right)^{\frac{p-1}{2}} \alpha(p) k_{\mathrm{e}} B^{\frac{p+1}{2}} \epsilon^{-\frac{p+1}{2}}$,

where $\alpha(p)$ is a combination of $\Gamma$ - functions (see Eq. (4.60) of Blumenthal \& Gould 1970). Equation (A.2) holds for photon energies far from the low- and high-energy ends of the spectrum $-\epsilon_{\mathrm{s}} \gamma_{\min }^{2}$ and $\epsilon_{\mathrm{s}} \gamma_{\max }^{2}$ respectively, where $\epsilon_{\mathrm{s}}=\frac{e B}{2 \pi m_{\mathrm{e}} c}$. The number density of synchrotron photons in the source is given by

$n_{\mathrm{s}}(\epsilon, r)=t_{R} \frac{\mathrm{d} N_{\mathrm{s}}}{\mathrm{d} \epsilon \mathrm{d} t}=\tilde{n}_{\mathrm{s}}(r) \epsilon^{-\frac{p+1}{2}}$

where $t_{R}$ is the crossing time of the source and $\tilde{n}_{\mathrm{s}}$ is the energyindependent factor on the right hand side of Eq. (A.2). The dependence on radius comes from the quantities $k_{\mathrm{e}}$ and $B$. The explicit functional form of the photon density can be found in Gould (1979). However, for the calculation of the Compton synchrotron logarithm we can safely proceed using the spatial averaged photon density

$\bar{n}_{\mathrm{s}}(\epsilon)=\left\langle\tilde{n}_{\mathrm{s}}\right\rangle \epsilon^{-\frac{p+1}{2}}$,

where $\left\langle\tilde{n}_{\mathrm{s}}\right\rangle=\frac{3}{R^{3}} \int \mathrm{d} r r^{2} \tilde{n}_{\mathrm{s}}(r)$. We assume further that the inverse Compton emissivity is given by a $\delta$ - function centered at the mean energy of an upscattered synchrotron photon of energy $\epsilon$

$j_{\text {ic }}\left(\epsilon_{1} ; \gamma, \epsilon\right)=A \epsilon_{1} \delta\left(\epsilon_{1}-4 / 3 \gamma^{2} \epsilon\right)$

where $A$ is a normalization factor. Then the total SSC power per unit energy emitted is found by

$J_{\text {ic }}\left(\epsilon_{1}\right)=\int \mathrm{d} \epsilon n_{\mathrm{s}}(\epsilon, r) \int \mathrm{d} \gamma N_{\mathrm{e}}(\gamma) j_{\text {ic }}\left(\epsilon_{1} ; \gamma, \epsilon\right)$,

In the above equation $N_{\mathrm{e}}=K_{\mathrm{e}} \gamma^{-p}$ is the total number of electrons per Lorentz factor $\gamma$ in the source. The normalization factor $K_{\mathrm{e}}$ is related to $k_{\mathrm{e}}$ through the integral $4 \pi \int \mathrm{d} r r^{2} k_{\mathrm{e}}(r)$. In order to simplify even more the calculation of the integral in (A.6) we use the average photon density

$J_{\mathrm{ic}}\left(\epsilon_{1}\right)=A K_{\mathrm{e}}\left\langle\tilde{n}_{\mathrm{s}}\right\rangle \int_{\epsilon_{\min }}^{\epsilon_{\max }} \mathrm{d} \epsilon \epsilon^{-\frac{p+1}{2}} I\left(\epsilon_{1}, \epsilon\right)$,

where $\epsilon_{\min }, \epsilon_{\max }$ are the effective minimum and maximum energies of the synchrotron photons and

$$
\begin{aligned}
I\left(\epsilon_{1}, \epsilon\right) & =\int_{\tilde{\gamma}_{\min }}^{\tilde{\gamma}_{\max }} \mathrm{d} \gamma \gamma^{-p} \epsilon_{1} \delta\left(\epsilon_{1}-\frac{4}{3} \gamma^{2} \epsilon\right) \\
& =\frac{1}{2}\left(\frac{\sqrt{3}}{2}\right)^{-p+1} \epsilon_{1}^{-\frac{p-1}{2}} \epsilon^{\frac{p-1}{2}}
\end{aligned}
$$

for $\frac{4}{3} \tilde{\gamma}_{\min }^{2} \epsilon<\epsilon_{1}<\frac{4}{3} \tilde{\gamma}_{\max }^{2} \epsilon$. Strictly speaking, the integral equals to zero for any other value of $\epsilon_{1}$. However, if one uses the complete expression of the inverse Compton emissivity, one finds that outside this energy range the intensity is highly reduced, but not actually zero. The lower limit of integration in (A.8) is determined by the kinematics of the compton scattering and the lower cutoff of the electron distribution, i.e.,

$\tilde{\gamma}_{\min }=\max \left[\gamma_{\min },\left(3 \epsilon_{1} / 4 \epsilon\right)^{1 / 2}\right]$.

The upper limit of integration is given by

$\tilde{\gamma}_{\max }=\min \left[\gamma_{\max }, \mathrm{m}_{\mathrm{e}} \mathrm{c}^{2} / \epsilon\right]$,

which takes into account the effect of the Klein-Nishina cutoff. For reasons of simplicity we proceed to the calculation of the SSC spectrum assuming that $\tilde{\gamma}_{\min }=\gamma_{\min }$ and $\tilde{\gamma}_{\max }=\gamma_{\max }$. 


\section{Appendix B: The Compton-synchrotron logarithm}

The integration over the synchrotron photon distribution (see Eqs. (A.7), (A.8)) leads to a factor

$\ln \Sigma=\ln \left(\frac{\epsilon_{\max }}{\epsilon_{\min }}\right)$

called the Compton synchrotron logarithm (Gould 1979). This quantity takes into account the effective minimum and maximum energies of synchrotron photons, which contribute to the principal branch of the SSC spectrum, i.e., to upscattered photons with energies between $\sim \epsilon_{\mathrm{s}} \gamma_{\min }^{4}$ and $\sim \epsilon_{\mathrm{s}} \gamma_{\max }^{4}$. The actual extent of the synchrotron power law segment is

$\epsilon_{\mathrm{s}} \gamma_{\min }^{2}<\epsilon<\epsilon_{\mathrm{s}} \gamma_{\max }^{2}$

where $\epsilon_{\mathrm{S}}=\frac{e B}{2 \pi m_{\mathrm{e}} c}$. In the previous section we showed that only for energies of the upscattered synchrotron photons between

$\frac{4}{3} \gamma_{\min }^{2} \epsilon<\epsilon_{1}<\frac{4}{3} \gamma_{\max }^{2} \epsilon$

the SSC spectrum differs significantly from zero. From Eqs. (B.2), (B.3) we find $\epsilon<\epsilon_{\mathrm{s}} \gamma_{\max }^{2}$ and $\epsilon<3 \epsilon_{1} / 4 \gamma_{\min }^{2}$.

1. If $3 \epsilon_{1} / 4 \gamma_{\min }^{2}<\epsilon_{\mathrm{s}} \gamma_{\max }^{2}$ then one sets

$$
\begin{aligned}
\epsilon_{\max } & =3 \epsilon_{1} / 4 \gamma_{\text {min }}^{2} \\
\epsilon_{\min } & =\epsilon_{\mathrm{s}} \gamma_{\text {min }}^{2} .
\end{aligned}
$$

Thus,

$$
\Sigma=\frac{3 \epsilon_{1}}{4 \epsilon_{\mathrm{s}} \gamma_{\min }^{4}}, \quad \frac{4}{3} \epsilon_{\mathrm{s}} \gamma_{\min }^{4}<\epsilon_{1}<\frac{4}{3} \epsilon_{\mathrm{s}} \gamma_{\min }^{2} \gamma_{\max }^{2} .
$$

2. If $3 \epsilon_{1} / 4 \gamma_{\min }^{2}>\epsilon_{\mathrm{s}} \gamma_{\max }^{2}$ then

$$
\epsilon_{\max }=\epsilon_{\mathrm{s}} \gamma_{\max }^{2} \text {. }
$$

However, synchrotron photons with energy $\epsilon_{\mathrm{s}} \gamma_{\min }^{2}$ cannot be upscattered to energies $\epsilon_{1}>\frac{4}{3} \epsilon_{\mathrm{s}} \gamma_{\min }^{2} \gamma_{\max }^{2}$. In this case the effective minimum energy of the synchrotron photons is given by

$\epsilon_{\min }=\frac{3 \epsilon_{1}}{4 \gamma_{\max }^{2}}$.

Thus we find

$$
\Sigma=\frac{4 \epsilon_{\mathrm{s}} \gamma_{\max }^{4}}{3 \epsilon_{1}}, \quad \frac{4}{3} \epsilon_{\mathrm{s}} \gamma_{\min }^{2} \gamma_{\max }^{2}<\epsilon_{1}<\frac{4}{3} \epsilon_{\mathrm{s}} \gamma_{\max }^{4} .
$$

Summarizing,

$\epsilon_{1} J_{\text {ic }} \propto \epsilon_{1}^{-\frac{(p-3)}{2}}\left\{\begin{array}{l}\ln \left(\frac{3 \epsilon_{1}}{4 \epsilon_{\mathrm{s}} \gamma_{\min }^{4}}\right), \frac{4}{3} \epsilon_{\mathrm{s}} \gamma_{\text {min }}^{4}<\epsilon_{1}<\frac{4}{3} \epsilon_{\mathrm{s}} \gamma_{\text {min }}^{2} \gamma_{\max }^{2} \\ \ln \left(\frac{4 \epsilon_{\mathrm{s}} \gamma_{\max }^{4}}{3 \epsilon_{1}}\right), \frac{4}{3} \epsilon_{\mathrm{s}} \gamma_{\text {min }}^{2} \gamma_{\max }^{2}<\epsilon_{1}<\frac{4}{3} \epsilon_{\mathrm{s}} \gamma_{\text {max }}^{4}\end{array}\right.$
The function above has a peak at the characteristic energy

$\epsilon_{\text {peak }}=\frac{4}{3} \epsilon_{\mathrm{s}} \gamma_{\min }^{2} \gamma_{\max }^{2}$

\section{References}

Barthelmy, S. D., Cannizzo, J. K., Gehrels, N., et al. 2005, ApJ, 635, L133 Blandford, R. D., \& McKee, C. F. 1976, Phys. Fluids, 19, 1130

Butler, N. R., \& Kocevski, D. 2007, ApJ, 668, 400

Dermer, C. D. 2007, ApJ, 664, 384

Dermer, C. D., \& Chiang, J. 1998, New Astron., 3, 157

Dermer, C. D., Böttcher, M., \& Chiang, J. 2000, ApJ, 537, 255

Eichler, D., \& Granot, J. 2006, ApJ, 641, L5

Evans, P. A., Beardmore, A. P., Page, K. L., et al. 2009, MNRAS, 397, 1177

Evans, P. A., Willingale, R., Osborne, J. P., et al. 2010, A\&A, 519, A102

Fan, Y., \& Piran, T. 2006, MNRAS, 369, 197

Fan, Y., Piran, T., Narayan, R., \& Wei, D. 2008, MNRAS, 384, 1483

Fenimore, E. E., Epstein, R. I., \& Ho, C. 1993, A\&AS, 97, 59

Ghisellini, G. 2008, in AIP Conf. Ser. 1000, ed. M. Galassi, D. Palmer, \& E. Fenimore, 448

Ghisellini, G., Ghirlanda, G., Nava, L., \& Firmani, C. 2007, ApJ, 658, L75

Giannios, D., \& Spitkovsky, A. 2009, MNRAS, 400, 330

Gould, R. J. 1979, A\&A, 76, 306

Granot, J., \& Kumar, P. 2006, MNRAS, 366, L13

Granot, J., \& Sari, R. 2002, ApJ, 568, 820

Granot, J., Königl, A., \& Piran, T. 2006, MNRAS, 370, 1946

Liang, E., Zhang, B., \& Zhang, B. 2007, ApJ, 670, 565

Lithwick, Y., \& Sari, R. 2001, ApJ, 555, 540

Mastichiadis, A., \& Kazanas, D. 2009, ApJ, 694, L54

Mastichiadis, A., \& Kirk, J. G. 1995, A\&A, 295, 613

Mészáros, P., \& Rees, M. J. 1997, ApJ, 476, 232

Nousek, J. A., Kouveliotou, C., Grupe, D., et al. 2006, ApJ, 642, 389

Paczynski, B., \& Rhoads, J. E. 1993, ApJ, 418, L5

Panaitescu, A., \& Kumar, P. 2000, ApJ, 543, 66

Panaitescu, A., \& Mészáros, P. 1998, ApJ, 501, 772

Panaitescu, A., Mészáros, P., Burrows, D., et al. 2006, MNRAS, 369, 2059

Pe'er, A., \& Waxman, E. 2004, ApJ, 613, 448

Petropoulou, M., \& Mastichiadis, A. 2009, A\&A, 507, 599

Piran, T. 1999, Phys. Rep., 314, 575

Piran, T. 2004, Rev. Mod. Phys., 76, 1143

Press, W. H., Teukolsky, S. A., Vetterling, W. T., \& Flannery, B. P. (ed.) 1992, Numerical recipes in FORTRAN, The art of scientific computing

Rees, M. J., \& Mészáros, P. 1992, MNRAS, 258, 41P

Sari, R., \& Esin, A. A. 2001, ApJ, 548, 787

Sari, R., Piran, T., \& Narayan, R. 1998, ApJ, 497, L17

Schady, P., Mason, K. O., Page, M. J., et al. 2007, MNRAS, 377, 273

Shao, L., Dai, Z. G., \& Mirabal, N. 2008, ApJ, 675, 507

Sironi, L., \& Spitkovsky, A. 2009, ApJ, 707, L92

Spitkovsky, A. 2008, ApJ, 682, L5

Tagliaferri, G., Goad, M., Chincarini, G., et al. 2005, Nature, 436, 985

Vaughan, S., Goad, M. R., Beardmore, A. P., et al. 2006, ApJ, 638, 920

Wijers, R. A. M. J., \& Galama, T. J. 1999, ApJ, 523, 177

Zhang, B., Fan, Y. Z., Dyks, J., et al. 2006, ApJ, 642, 354

Zhang, B., Liang, E., \& Zhang, B. 2007, ApJ, 666, 1002 